\title{
Physical Activity Levels in Children with Sensory problems: Cross-sectional Analyses from the Millennium Cohort Study
}

Background: Hearing and vision impairments/problems are associated with increased risk of premature mortality in adulthood. One potential pathway explaining this association is reduced levels of physical activity. Reductions in activity levels due to sensory impairments may commence earlier in life; however, associations between sensory impairments/problems and activity levels in childhood are not well understood.

Objective: The objective of this study was to examine associations between hearing and vision problems and objectively measured activity levels in a representative sample of British children.

Methods: Data were drawn from sweep 4 of the Millennium Cohort Study, a prospective cohort study among children aged 7. Child hearing and vision problems were reported by parents in a postal survey. Children were also invited to have their physical activity measured objectively via accelerometry. A total of 6,410 children had valid accelerometry data accompanied by complete survey data on the variables of interest. The main outcomes were objectively measured moderate-to-vigorous physical activity, steps and sedentary time. Adjusted linear regression was used to examine associations between vision and hearing problems and objectively measured activity levels.

Results: In this sample of 7-year old children, $16.7 \%(\mathrm{n}=1068)$ had a reported vision problem and $11.6 \%(n=745)$ had a hearing problem. Reported vision problems in both eyes, but not one eye, was associated with 2 fewer minutes of moderate-to-vigorous physical activity per day (B=-2.1 95\% Confidence Intervals [CI] -3.9 to -0.4) and almost 200 fewer steps per day ( $B=-198.495 \% \mathrm{CI}$, -398.4 to 1.6). Hearing problems were not associated with activity levels in either one or both ears.

Conclusion: Children with visual problems affecting both eyes, but not one eye, are likely to have lower levels of physical activity. Strategies to promote physical activity in children with visual problems are warranted. 


\section{Introduction}

Physical inactivity (defined here as not meeting physical activity guidelines) is a major risk factor in the development of cardiometabolic disease, osteoporosis, and poor mental health (1-5). An important factor in physical activity is that of life-long engagement, supporting lifelong health, and crucially, an increased likelihood of physical activity into older age (6-8). National government guidelines (9) suggest children should engage in "moderate to vigorous intensity physical activity (MVPA; activities that get one moving fast enough or strenuously enough to burn off three to six times as much energy per minute as one does when sitting quietly) for at least 60 minutes and up to several hours every day” for good health. However, physical activity levels among children in the UK are low, with reports suggesting that one third of children do not meet government recommendations (10). Understanding the barriers to physical activity in childhood to facilitate effective physical activity promotion is essential.

Correlates of physical activity in children are well documented (11-12). Importantly, low levels of physical activity are known to be a greater problem in children with disabilities (1314). Sensory problems, such as vision and hearing impairments may be key barriers to engagement in physical activity in children. The WHO states that in Europe 31.7 thousand people per million population are visually impaired, 28.7 have low vision and 3 are blind (15) while 32 million children across the world live with disabling hearing loss.

Limited research has explored the association with eyesight problems and physical activity. However, research has suggested that children with severe visual impairment are less physically active than their sighted counterparts $(14,16-19)$. However, this literature is limited, precluding any firm conclusion, owing to small sample sizes or subjective measures of activity behaviour. For example, parents reporting child physical activity may overstate 
actual activity levels owing to fears of being judged, or indeed may misinterpret what constitutes light, moderate or vigorous activity. Houwen, Hartman and Visser (17) found that, among a city-wide Dutch sample of ninety-six children with and without visual impairment aged 6-12 years, total activity, and participation in MVPA was significantly lower for the children with visual impairment, while time spent in sedentary and light behaviour was significantly higher. In addition, body mass index (BMI), percentage body fat, and locomotor and object control were all associated with degree of visual impairment and level of physical activity. Findings from this study are however unlikely to generalize to the UK population owing to the small sample size of children residing in the Dutch city under study. Further research is required in UK population-based samples. Nevertheless, children with visual impairment may be a group at risk of the aetiology of diseases associated with inactivity.

Perceived sociological barriers to physical activity for children with visual impairment have been reported as parental support and parents' physical activity, lack of security, motivation, professional training and information about activities (16). From a physical perspective, children with sight impairment are less likely than their peers to feel able to carry out tasks, play organised sport, take part in physical activities, make journeys on foot outside of school on their own or with other children, and are more likely to feel tired at school all or most of the time, leading to higher inactivity (18). Furthermore, older children who are visually impaired are less active than their younger counterparts when entering the second decade of life $(14,18,19)$; highlighting the need to explore this disability as an age specific correlate of physical activity. Importantly, to date no one has investigated whether the extent of visual impairment (i.e. whether in one or both eyes) has a detrimental influence on levels of freeliving physical activity. Further research is required in this area.

Hearing impairment and reduced physical activity has been reported widely in older adults (see e.g. 20). Examining the physical activity levels of deaf and hearing impaired children 
and sociodemographic correlates (21-23), research suggests that deaf children demonstrate similar or slightly lower fitness levels when compared to those with good hearing $(21,22,24-$ 27). However, research is yet to explore the physical activity levels of hearing impaired children in population based samples, despite knowledge that disability is associated with increased levels of effort and higher levels of fatigue, which lead to greater inactivity.

Hearing and vision impairments are associated with increased risk of mortality in adulthood (28). One potential pathway explaining this association is reduced levels of physical activity across the lifespan $(29,30)$. Reductions in activity levels due to sensory impairments may commence earlier in life; however, the associations between sensory impairments and activity levels in childhood is not well understood. The aim of the present study was to examine associations between hearing and vision problems and objectively measured activity levels in a large representative sample of 7-year old British children. We hypothesized that eyesight or hearing problems will be associated with low levels of physical activity.

\section{Methods}

The Millennium Cohort Study (MCS) is a prospective study of a nationally representative UK sample of children born at the turn of the century (between September 2000 and January 2002). Eligible children were identified from the records of Child Benefit. Information was collected on 18,818 children at 9 months of age from 1 parent (usually the child's mother). Further surveys were administered at the ages of 3, 5 and 7 years. All measures were collected in the child's home. To maximize sample size the present manuscript used data from the age 7 survey only where physical activity was objectively measured using waist worn accelerometers (Actigraph GT1M, Pensacola, Florida). Ethical approval was granted by the South West and London Multi-Centre Research Ethics Committees. 


\section{Exposure Variables}

Parents (predominantly mothers) reported whether child "ever had any problems with [his/her] eyesight or eyes” (yes/no); if yes “does or did it affect one or both eyes” (one/both); whether child "ever had any problems with [his/her] hearing or ears” (yes/no); if yes “does or did it affect one or both ears”. Children were classified as having no eyesight problems, eyesight problem in one eye, eyesight problem in both eyes, no hearing problems, hearing problems in one ear and hearing problem in both ears. It is important to note that the measure for visual problems used in the present study may encompass those with blindness (full or partial), eye injury, and those with 20/20 vision with correctives. The same principle applies to the measure relating to hearing problems.

\section{Outcome Variables}

Physical activity, steps and sedentary time were measured objectively using Actigraph GT1M accelerometers when participants were 7 years of age (between May 2008 and August 2009). Actigraph accelerometers are a valid and reliable way to measure physical activity in young people. Full details on the accelerometry procedures have been published previously (31). In brief, accelerometers were delivered by mail to consenting participants and programmed to record data at 15-second intervals (15-second epoch length). Participants were instructed to wear the accelerometers around their waists during waking hours, and to take them off during water-based activities, for 7 consecutive days. Devices were returned and information was downloaded using Actigraph software. A total of 6,675 children (3,176 boys) met the inclusion criteria, which was set as having at least 2 days with 10 hours or more of wear time. 
Time spent engaging in physical activities of varying intensities was derived using cutpoints generated from a prior calibration study in 7-year-old children. Specifically, time sedentary was classified as fewer than 100 counts per minute, and time in MVPA was classified as more than 2,241 counts per minute. All outcome measures were approximately normally distributed. Thus, no transformations were required.

\section{Covariates}

Parents/ carers (usually mothers) reported child(ren)'s sex, age, ethnicity (classified as White British or Other) and income (classified as above or in poverty). Children were measured by trained interviewers for height and weight from which body mass index (BMI) was calculated using standard formulae. Children were classified as not overweight or overweight/obese. Total actigraph wear time across the week was also recorded.

\section{Analyses}

Characteristics of the study population were analysed using descriptive statistics. As our outcomes were continuous, adjusted (for pre-specified covariates) linear regression was used to examine associations between ever having vision and hearing problems and objectively measured daily physical activity levels (MVPA, steps, sedentary). Models were adjusted for age, sex, ethnicity, poverty status, BMI status and accelerometry wear time. Residual plots confirmed that residuals were normally distributed and that the homoscedasticity assumption was not violated. Variance inflation factors did not exceed 2, suggesting low possibility of multicollinearity. 


\section{Results}

The final sample consisted of 6,410 children with valid accelerometry data and complete parent-reported questionnaire data. Compared to the analytic sample, those excluded from the analysis due to incomplete/invalid accelerometry data, but who had complete questionnaire data ( $n=6698)$, were more likely to be in poverty ( $21.6 \%$ vs. $35.4 \%, p<0.001)$, were more likely to be overweight or obese $(17.3 \%$ vs. $23.9 \% \mathrm{p}<0.001)$ and were less likely to be White British (88.9\% vs. 81.7\%, p<0.001). However, children excluded from the analysis did not differ from the final sample in terms of visual and hearing problems. In the final sample, 16.7\% $(n=1068)$ had a visual problem and $11.6 \%(n=745)$ had a hearing problem (Table 1).

\section{Insert Table 1 here}

Visual problems in both eyes, but not one eye, were associated with 2 fewer minutes of moderate-to-vigorous physical activity per day $(B=-2.195 \%$ Confidence Intervals [CI -3.9 to -0.4; Table 2). The association between visual problems in both eyes and steps approached significance ( $\mathrm{B}=-198.495 \% \mathrm{CI}$, $(-398.4$ to 1.6$)$. There were no associations between visual problems and sedentary time. Hearing problems were not associated with activity levels in either one or both ears (Table 3).

Insert Table 2 and 3 here 


\section{Discussion}

To the best of our knowledge, this is the first study to investigate the association between parents reported vision and hearing of their children and objectively measured physical activity behaviour in a large general population of children in the UK.

In this sample of 7-year old children, $16.7 \%(n=1068)$ had a reported vision problem and $11.6 \%(n=745)$ had a hearing problem. Reported vision problems in both eyes, but not one eye, were associated with 2 fewer minutes of moderate-to-vigorous physical activity per day and almost 200 fewer steps per day. Hearing problems were not associated with activity levels. The findings of this study support wider research that suggests children with visual problems have lower physical activity levels then their sighted counterparts (17). On the other hand, hearing problems are not as strongly correlated with physical activity (21). Many of the covariates reported in this analysis (i.e. age, sex, ethnicity, poverty status, BMI status) have been related to physical activity in previously published work $(11,12)$. That associations between vision and physical activity remained robust after accounting for these important confounders highlights the importance of this sensory deficit as a barrier to physical activity. These findings support and add to previous work that has identified lower levels of physical activity in smaller samples with more severe visual impairment, often utilising self-reported activity measures (14, 16-19).

The prevalence of lower levels of MVPA in children with visual problems in both eyes is likely linked to their perceived ability to carry out tasks, play organised sport, and take part in physical activities, as well as their predisposition to feel tired at school all or most of the time (18). At a basic level, vision problems in both eyes likely presents a barrier to the speed and visual acuity required to perform MVPA movements such as skipping, sprinting and football 
(32). Fewer steps per day could be a result of less journeys taken on foot, outside of school or on their own (18).

Poor eyesight is related to lower levels of physical activity in older adults (20), which in turn increases risk of diseases such as type 2 diabetes that is associated with further visual problems manifesting throughout the lifespan (33). It is important to intervene in this selfperpetuating barrier in childhood activity levels to reduce disease risk and increase quality of life in those with visual problems.

The percentage of children with visual problems is higher in the present study than reported previously $(15,34)$. The present measure of visual problems is broad in comparison to measures recording specific eye conditions or visual impairment severity. Thus, the measure here encapsulates a greater variety of possible visual problems. In addition, parents may think that needing spectacles suggests that their child has a visual problem even though the child attains very good vision with their spectacles.

The current analysis is cross-sectional in design; therefore, the causal direction of the observed association cannot be inferred. It is unclear whether poor vision caused lower level of physical activity, or whether lower levels of physical activity caused poor vision. However, given the age of the children, it is more likely that poor vision caused lower levels of physical activity, since increased likelihood of cardiometabolic diseases that lead to poor eyesight such as diabetes do not manifest until later in life.

Hearing problems were not associated with lower levels of physical activity in children, which is a finding in line with wider previous literature (21-23). However, to the authors' knowledge, this is the first study to examine objectively measured physical activity levels of children with hearing problems in a large UK based population study. The results suggest that hearing problems are not key barriers to physical activity. It is important to examine the 
progression of fitness and physical activity of children with hearing problems throughout the lifespan, and into old age, where sensory deficits are again associated with lower levels of physical activity (20).

A clear strength of the present study is the large and representative sample and the use of objective measures of physical activity. Data from this study must be interpreted with caution. The measure of disability in the present study refers to any retrospective vision or hearing problem and physical activity was measured at age 7 only. There is a possibility that children no longer had visual or hearing problems at the time of activity measurement. Therefore, our findings may under report the true difference in activity levels between those with visual or hearing problems and those without. Further research is needed that more clearly discern current vision/ hearing problems versus past vision/ hearing problems versus no vision/ hearing problems. Another limitation is that accelerometers only provided data on a single week, which may not be a true reflection of typical behaviour.

In conclusion, the current study suggests that children with reported visual problems in both eyes, but not one eye, are likely to have lower levels of physical activity. Strategies to promote physical activity in children with visual problems are warranted.

\section{References}

1. Department of Health: Choosing activity: a physical activity action plan. 2005, London: Stationery Office

2. Flynn M, McNeill D, Maloff B, Mutasingwa D, Wu M, Ford C, Tough S. Reducing obesity and related chronic disease risk in children and youth: a synthesis of evidence with 'best practice' recommendations. Obesity Reviews; 2006, 7: 7-66.

3. Biddle S, Gorely T, Stensel D. Health-enhancing physical activity and sedentary behaviour in children and adolescents. Journal of Sports Sciences; 2004, 22: 679-701.

4. Andersen L, Harro M, Sardinha L, Froberg K, Ekelund U, Brage S, Anderssen S. Physical activity and clustered cardiovascular risk in children: a cross-sectional study (The European Youth Heart Study). Lancet; 2006, 368: 299-304. 
5. Korczak DJ, Madigan S, Colasanto M. Children's physical activity and depression a metaanalysis. Pediatrics; 2017, 139(4). DOI: 10.1542/peds.2016-2266.

6. Smith L, Gardner B, Hamer M. Childhood correlates of adult TV viewing time: a 32-year follow-up of the 1970 British Cohort Study. J Epidemiol Community Health; 2015, 69:30913.

7.Smith L, Gardner B, Aggio D, Hamer M. Association between participation in outdoor play and sport at 10 years old with physical activity in adulthood. Prev Med; 2015, 74:31-5.

8. Smith L, Fisher A , Hamer M. Prospective association between objective measures of childhood motor coordination and sedentary behaviour in adolescence and adulthood. Int $J$ Behav Nutr Phys Act; 2015, 12:75.

9. Department of Health: UK physical activity guidelines. 1 July 2011: https://www.gov.uk/government/publications/uk-physical-activity-guidelines

10. Department of Health: Health Survey for England 2002: The Health of Children and Young People. 2003, London: Stationery Office.

11. Sallis J.F, \& Hovell M.F. Determinants of exercise behavior. Exercise and Sport Sciences Review; 1990, 18: 307-330.

12. Sallis J.F, Simons-Morton B.G, Stone E.J, Corbin C.B, Epstein L.H, Faucette N, Iannotti R.J, Killen J.D, Klesges R.C, Petray C.K, Rowland T.W, \& Taylor W.C. Determinants of physical activity and interventions in youth. Medicine and Science in Sports and Exercise; 1992, 24: S248-S257.

13. Shields N. Synnot A.J. Barr M. Perceived barriers and facilitators to physical activity for children with disability: a systematic review. Bri Joun. Sports Med; 2012, 46(14): 989-97.

14. Langmuir P.E. and Bar-Or O. Factors Influencing the Physical Activity Levels of Youths With Physical and Sensory Disabilities. Adapted Physical Activity Quarterly; 2000, 17(1), 40-54.

15. The World Health Organization, Global Data on Visual Impairments 2010

16. Greguol M1, Gobbi E, Carraro A. Physical activity practice, body image and visual impairment: a comparison between Brazilian and Italian children and adolescents. Res Dev Disabil; 2014, 35(1):21-6.

17. Houwen S, Hartman E, and Visser C. Physical activity and motor skills in children with and without visual impairments. Med Sci for Sports and Exerc; 2009, 41(1):103-109.

18. Harris J, Keil S, Lord C, Lloyd C.L. Sight impairment at age eleven: secondary analysis of the Millennium Cohort Survey. RLSB, RNIB and NatCen; 2014.

19. Oh HK, Ozturk MA, Kozub, FM. Physical Activity and Social Engagement Patterns During Physical Education of Youth with Visual Impairments. ProQuest; 2004, 36(1): 39-49.

20. Smith L, Timmis M.A, Pardhan S, Latham K, Johnstone J, Hamer, M. Physical inactivity in relation to self-rated eyesight: cross-sectional analysis from the English Longitudinal Study of Ageing. BMJ Open Ophthamology; 2017, 1:e000046.

21. Ellis M K, Lieberman L J, Fittipauldi-Wert J, Dummer G M. Health-related physical fitness of deaf children: How do they measure up? Palaestra; 2005, 21: 36-43.

22. Dair J, Ellis M K, Lieberman L J. Prevalence of overweight among deaf children. American Annals of the Deaf; 2006, 151: 318-326. 
23. Ellis KM, Lieberman L.J, Dummer G.M. Parent Influences on Physical Activity Participation and Physical Fitness of Deaf Children. J Deaf Stud Deaf Educ; 2014, 19(2): 270-281.

24. Ellis M K. Influence of parental hearing levels and school placement on the healthrelated physical fitness and community sports involvement of children who are deaf. Palaestra; 2001, 17: 44-49.

25. Ellis M K, Butterfield S, Lehnhard R. Grip-strength performances by 6 to 19-year-old children with and without hearing impairments. Perceptual and Motor Skills; 2000, 90: 279282.

26. Goodman J. Hopper C. Hearing impaired children and youth: A review of psychomotor behavior. Adapted Physical Activity Quarterly; 1992, 9: 214-236.

27. Lieberman L J, and McHugh E. Health-Related Fitness of Children Who Are Visually Impaired. Journal of visual impairment and blindness; 2001, 95(5).

28. Rajala U, Pajunpaa H, Koskela P, et al. High cardiovascular disease mortality in subjects with visual impairment caused by diabetic retinopathy. Diabetes Care; 2000, 23:957-61.

29. Smith L, Gardner B, Fisher A, Hamer M. Patterns and correlates of physical activity behaviour over 10 years in older adults: prospective analyses from the English Longitudinal Study of Ageing. BMJ Open; 2015, 5:e007423.

30. Public Health England. Everybody active every day. An evidence based approach to physical activity. UK: Public Health England, 2014.

31. Griffiths L, Cortina-Borja M, Sera F, Pouliou T, Geraci M, Rich C, Cole T, Law C, Joshi H, Ness A, Jebb S, Dezateux C. How active are our children? Findings from the Millennium Cohort Study. BMJ Open. 3(8)

32. Winnick, J. P. Performance of visually impaired youngsters in physical education activities: Implications for mainstreaming. Adapted Physical Activity Quarterly; 1985, 3: 5866.

33. Drivsholm, T. Symptoms, signs and complications in newly diagnosed type 2 diabetic patients, and their relationship to glycaemia, blood pressure and weight. Diabetologia; 2005, 48(2), 210.

34. Royal National Institute of Blind People, Children and young people with vision impairment: who's counting? 2014 
Table 1. Descriptive characteristics of sample $(n=6410)$

\begin{tabular}{lll}
\hline & $\%(\mathrm{n})$ & Mean (SD) \\
\hline Age (years) & & $7.2(2.4)$ \\
Male & $48.9(3133)$ & \\
White British race/ethnicity & $88.9(5699)$ & \\
Poverty-level income & $21.6(1384)$ & \\
Overweight/obese & $17.3(1110)$ & \\
Sedentary time (min/day) & & $393.2(66.8)$ \\
Steps (per/day) & & $10229.3(2466.7)$ \\
MVPA (min/day) & & $62.3(22.4)$ \\
Total 7-day wear time (min) & & \\
Visual problems & & \\
$\quad$ None & $83.3(5342)$ & \\
$\quad$ One eye & $7.3(465)$ & \\
$\quad$ Both eyes & $9.4(603)$ & \\
Hearing problems & & \\
$\quad$ None & $88.4(5665)$ & \\
$\quad$ One ear & $4.4(281)$ & \\
Both ears & $7.2(464)$ & \\
\hline
\end{tabular}

Table 2. Associations between vision problems and objectively measured activity levels $(n=6410)$

\begin{tabular}{lccc}
\hline \multicolumn{4}{c}{ B $(95 \%$ Confidence Intervals $)$} \\
& Sedentary time & Steps & MVPA \\
\hline No vision problem & REF & REF & REF \\
Vision problem in one eye & $-1.1(-6.9,4.7)$ & $47.5(-177.4,272.3)$ & $-0.9(-2.9,1.1)$ \\
Vision problem in both eyes & $-0.4(-5.5,4.8)$ & $-198.4(-398.4,1.6)$ & $-2.1(-3.9,-0.4)$ \\
\hline
\end{tabular}

Adjusted for age, sex, ethnicity, poverty status, bmi status and accelerometry wear time 
Table 3. Associations between hearing problems and objectively measured activity levels ( $n=6410)$

\begin{tabular}{|c|c|c|c|}
\hline \multicolumn{4}{|c|}{ B (95\% Confidence Intervals) } \\
\hline & Sedentary time & Steps & MVPA \\
\hline No hearing problem & REF & REF & REF \\
\hline Hearing problem in one ear & $-5.5(-12.8,1.8)$ & $40.2(-244.4,324.7)$ & $-0.9(-3.4,1.6)$ \\
\hline Hearing problem in both ears & $-1.7(-7.4,4.1)$ & $-56.6(-281.6,168.4)$ & $-0.4(-2.4,1.6)$ \\
\hline
\end{tabular}

Adjusted for age, sex, ethnicity, poverty status, bmi status and accelerometry wear time 